\title{
Recessive mutations in the CYP4V2 gene in East Asian and Middle Eastern patients with Bietti crystalline corneoretinal dystrophy
}

\author{
J Lin, K M Nishiguchi, M Nakamura, T P Dryja, E L Berson, Y Miyake
}

J Med Genet 2005;42:e38 (http://www.jmedgenet.com/cgi/content/full/42/6/e38). doi: 10.1136/jmg.2004.029066

\begin{abstract}
Background: Bietti crystalline corneoretinal dystrophy (BCD) is an autosomal recessively inherited disorder characterised by tiny yellowish glittering retinal crystals, choroidal sclerosis, and crystals in the peripheral cornea, associated with progressive night blindness. CYP4V2, encoding a member of cytochrome p450 (CYP450) protein family, was recently identified as the causative gene.

Methods: We recruited 11 unrelated patients with $B C D$ and characteristic clinical features; eight of Japanese, two of Middle Eastern, and one of Chinese ancestry. Genomic DNA was extracted from peripheral blood leucocytes, and all 11 exons and the flanking intron splice sites of the CYP4V2 gene were amplified and sequenced. A complete ophthalmological examination was performed.

Results: We found recessive mutations in the CYP4V2 gene in all of the 11 patients. Two novel mutations, L173W and Q450X, were identified in a Japanese patient and two unrelated patients from Middle Eastern countries, respectively. Each patient was a homozygote. A previously reported mutation IVS6-8_810delinsGC was identified in seven unrelated Japanese patients and the Chinese patient with $B C D$. All patients with $B C D$ shared a characteristic fundus appearance with numerous intraretinal crystal deposits and atrophy of the retinal pigment epithelium. However, the clinical findings, including elecroretinograph recordings, indicated that there was considerable variation in the degree of visual dysfunction even among patients of similar ages carrying the same mutation.

Conclusions: Defects in CYP4V2 are the main cause of BCD. The IVS6-8_810delinsGC mutant allele may be especially prevalent among patients with BCD in East Asian countries, resulting from a single founder. Variation of disease severity suggests that environmental or additional genetic factors influence the course of the retinal disease.
\end{abstract}

B ietti crystalline corneoretinal dystrophy (BCD) is an autosomal recessively inherited disorder, which was first reported in 1937 by Bietti. ${ }^{1}$ It is characterised by multiple small glistening intraretinal crystalline deposits scattered throughout the posterior pole associated with progressive atrophy of the retinal pigment epithelium (RPE) and choroidal sclerosis. The crystalline deposits are also present in circulating lymphocytes, and in the limbal cornea in some patients. ${ }^{1-6}$ The disease typically manifests itself between the second and fourth decades of life with progressive night blindness, decreased visual acuity, and constriction of the visual fields. ${ }^{5}$ The disease progresses slowly, and the vision is severely impaired in the late stages. Although BCD is a relatively rare disease, the diagnosis is straightforward because of the characteristic clinical features. Patients with $\mathrm{BCD}$ have been reported from most parts of the world, but it appears to be more common in East Asian populations, especially in China and Japan. ${ }^{578}$

The gene responsible for $\mathrm{BCD}$ has been mapped to chromosome 4q35, ${ }^{9}$ and within this region CYP4V2, encoding a member of cytochrome p450 (CYP450) protein family, was recently identified as the causative gene. ${ }^{10}$ The gene contains 11 exons spanning $21.7 \mathrm{~kb}$, and is expressed in various tissues, most abundantly in the retina. ${ }^{10}$ The CYP450 protein family is a group of enzymes that use iron to oxidise various substrates, including potentially harmful substances, thereby making them more water soluble and promoting their biological processing. Some members of the CYP450 family are known to have a role in lipid metabolism, and it is possible that the protein encoded by CYP4V2 plays a role in processing lipids such as fatty acids or a role in steroid metabolism. ${ }^{10}$ This speculation is based on a published biochemical analysis of cultured lymphocytes from patients with $\mathrm{BCD}$, which showed abnormally high levels of triglycerides and cholesterol, and the absence of two fatty acid binding proteins. ${ }^{.1}$

There has been only one previous report of mutations in CYP4V2 in BCD. We undertook this study to better understand the range of pathogenic mutations and to search for possible non-allelic heterogeneity. Here we report our mutation screen of the CYP4V2 gene in 11 unrelated patients with BCD of East Asian or Middle Eastern ancestry and the clinical findings in the patients.

\section{PATIENTS AND METHODS}

This study was performed in accordance with the tenets of the Declaration of Helsinki, and was approved by the internal review boards at the Nagoya University Graduate School of Medicine, Massachusetts Eye and Ear Infirmary, and Harvard Medical School. For the study, 11 unrelated patients who had been diagnosed with BCD on the basis of their characteristic clinical features were recruited. Eight of the patients were of Japanese ancestry and were ascertained at the Department of Ophthalmology, Nagoya University Graduate School of Medicine. Two patients of Middle Eastern ancestry and one of Chinese ancestry were from the Berman-Gund Laboratory for the Study of Retinal Degenerations, Harvard Medical School. None of the parents or the children of the patients were affected, and five of the unrelated patients were the offspring of consanguineous relationships. The ophthalmological evaluations included the measurement of best corrected visual acuity, biomicroscopy, ophthalmoscopy, fundus photography, Goldmann kinetic perimetry, and electroretinograms (ERGs). Full field ERGs were recorded with the previously published methods using separate protocols at Nagoya University ${ }^{12}{ }^{13}$ and at Harvard Medical School. ${ }^{14-16}$ After informed consent was obtained, blood 
Family $270, m=L 173 \mathrm{~W}$
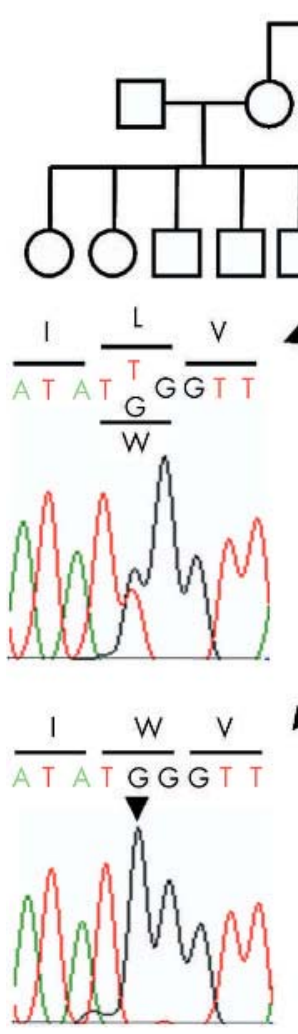

\section{(2)}

1 (1)
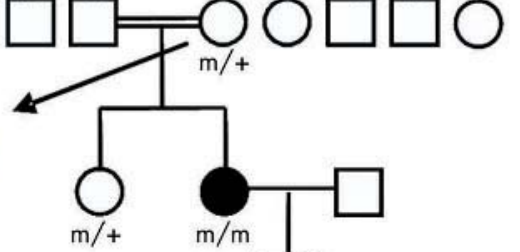

$\mathrm{m} / \mathrm{m}$
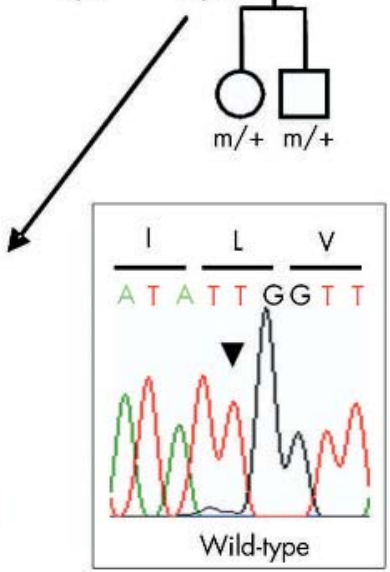

Figure 1 DNA sequence chromatogram and schematic pedigrees of two $\mathrm{BCD}$ patients (patient 270, left and patient 288-002, right) with novel mutations (L173W, left and Q450X, right) identified in this study. Affected patients are shown with solid symbols and unaffected with open symbols. Some birth orders are altered to preserve patient confidentiality. samples were taken from the patients and, in some cases, their relatives. Normal subjects without a history of a retinal disease were recruited as controls.

Genomic DNA was extracted from peripheral blood leukocytes using standard methods. The exons containing the open reading frame of the CYP4V2 gene and the flanking intron splice sites were individually amplified by PCR. ${ }^{10}$ The amplified DNA fragments were purified and directly sequenced using a dye terminator cycle sequencing kit (version 3.1; Applied Biosystems; Foster City, CA, USA) and
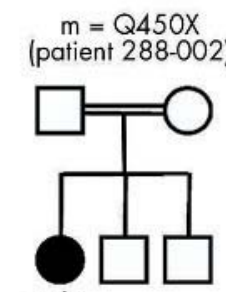

$\mathrm{m} / \mathrm{m}$
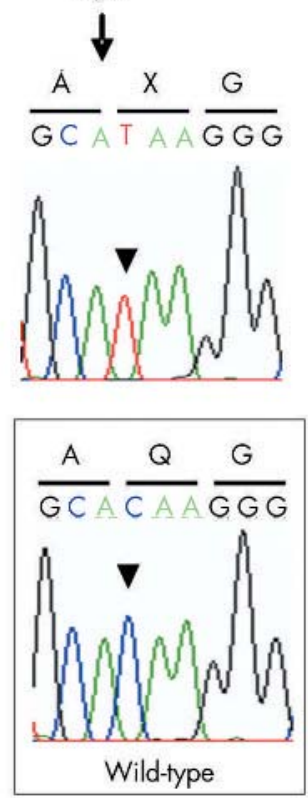

Table 1 Summary of mutations in CYP4V2 and clinical information in patients with BCD

\begin{tabular}{|c|c|c|c|c|c|c|c|c|}
\hline ID & Ethnicity & Nucleotide* & Protein & Age & Sex & $\operatorname{Va}(\mathbf{R}, \mathbf{L})$ & $\begin{array}{l}\text { †ERG } \\
\text { amplitudes } \\
R, L(\mu V)\end{array}$ & $\begin{array}{l}\text { Corneal } \\
\text { deposit }\end{array}$ \\
\hline 252 & Japanese & IVS6-8_810delinsGC & Skip exon $7 \S$ & 50 & $M$ & $1.0,0.6$ & $120, N D$ & \\
\hline 259 & Japanese & IVS6-8_810delinsGC & Skip exon $7 \S$ & 52 & $\mathrm{~F}$ & $0.5,1.0$ & ND, 27 & \\
\hline 260 & Japanese & IVS6-8_810delinsGC & Skip exon $7 \S$ & 46 & $\mathrm{~F}$ & $0.7,0.9$ & $207, \mathrm{ND}$ & + \\
\hline 265 & Japanese & IVS6-8_810delinsGC & Skip exon $7 \S$ & 38 & $\mathrm{~F}$ & $0.2,1.5$ & 274,296 & \\
\hline 272 & Japanese & IVS6-8_810delinsGC & Skip exon $7 \S$ & 52 & $M$ & $0.9,0.7$ & $385, \mathrm{ND}$ & + \\
\hline 303 & Japanese & IVS6-8_810delinsGC & Skip exon $7 \S$ & 54 & $M$ & $0.5,0.5$ & $311, \mathrm{ND}$ & + \\
\hline 312 & Japanese & IVS6-8_810delinsGC & Skip exon $7 \S$ & 47 & $\mathrm{~F}$ & $1.2,1.2$ & $312, \mathrm{ND}$ & + \\
\hline 270 & Japanese & $518 \mathrm{~T} \rightarrow \mathrm{G}$ & L173W & 54 & $\mathrm{~F}$ & $1.0,1.2$ & $296, N D$ & + \\
\hline $288-001$ & Chinese & $\begin{array}{l}\text { IVS6-8_810delinsGC } \\
992 A \rightarrow C\end{array}$ & $\begin{array}{l}\text { Skip exon7§ } \\
\text { H331P }\end{array}$ & 26 & $\mathrm{~F}$ & $0.5,0.6$ & 329,323 & + \\
\hline 288-002 & $\begin{array}{l}\text { Middle } \\
\text { Eastern }\end{array}$ & $1348 \mathrm{C} \rightarrow \mathrm{T}$ & Q450X & 21 & $\mathrm{~F}$ & $0.4,0.2$ & 229,206 & + \\
\hline $288-003$ & $\begin{array}{l}\text { Middle } \\
\text { Eastern }\end{array}$ & $1348 \mathrm{C} \rightarrow \mathrm{T}$ & Q450X & 45 & $M$ & $0.8,0.05$ & 71,29 & + \\
\hline \multicolumn{9}{|c|}{$\begin{array}{l}\text { ND, not determined. *All patients were homozygotes except } 288-001 \text {, who is a presumed compound } \\
\text { heterozygote; tb-wave amplitudes from dark-adapted single white flash ERGs extracting mixed rod and cone } \\
\text { responses. For the first eight patients (patients } 252-270 \text { ), ERGs were recorded at Nagoya University (normal range } \\
\geqslant 314 \mu \mathrm{V} \text { ) and, for the last three patients (patients } 288-001,288-002 \text {, and } 288-003 \text { ), at Harvard Medical Schoo } \\
\text { (normal range } \geqslant 350 \mu \mathrm{V} \text { ). §The predicted consequence of exon } 7 \text { skipping is in frame deletion of residues } \\
268-329 \text {. }\end{array}$} \\
\hline
\end{tabular}

ABI automated DNA sequencers (model 3100; Applied Biosystems). The numbering of the bases of the cDNA and genomic sequences in this article is according to Genbank accession numbers XM_209612 and NC 000004, respectively. Prediction of the structure of CYP4V2 was based on comparison and analysis of crystal coordinates from mammalian CYP2C5 (Protein Data Bank (PDB) ID 1dt6) and bacterial CYPBM3 (PDB ID 2hpd). ${ }^{17} 18$

Partial alignment of amino acid sequences of six eukaryotic CYP4V2 homologues selected using an online analysis tool

whw imedgenticom 


\begin{tabular}{|c|c|}
\hline & $\stackrel{L 173}{\downarrow}$ \\
\hline Human CYP4V2 & EQANI $\underline{L}$ VKKLE \\
\hline Mouse Cyp4v3 & EQANII LVNKLE \\
\hline Rat LOC361155 & EQANI $\underline{L} V N K L E$ \\
\hline Mosquito 1272634 & KQSYVLVRQLE \\
\hline Nematode $4 \mathrm{~m} 169$ & EQSKILVQKLC \\
\hline Thale cress at $3 g 25180$ & SEVNTLIKHLY \\
\hline
\end{tabular}

Figure 2 Partial amino acid sequence alignment surrounding the site of the L173W mutation in six eukaryotic CYP4V2 homologues: human CYP4V2 (NP_997235.1), mouse Cyp4v3 (NP_598730.1), rat LOC361155 (XP_341441.1), mosquito 1272634 (XP 311534.1), nematode $4 \mathrm{~m} 16 \overline{9}$ (NP_502152.2), and thale cress at3g25180 (NP_189154.1). An arrow indicates the position of the residue altered by the novel missense mutation L173W.

(www.ncbi.nlm.nih.gov/entrez/query.fcgi? $\mathrm{db}=$ homologene) were constructed and analysed. The proteins (Genbank accession numbers) used were human CYP4V2 (NP 997235.1), mouse Cyp4v3 (NP 598730.1), rat LOC36115 $\overline{5}$ (XP_341441.1), mosquito 1272634 (XP_311534.1), nematode $4 \mathrm{ml69}$ (NP_502152.2), and thale cress at3g25180 (NP_ $189154.1) .{ }^{10-21}$

\section{RESULTS}

We identified four different mutations, L173W (c.518T $\rightarrow G$ ), IVS6-8 810delinsGC, H331P $($ c.992A $\rightarrow$ C), and Q450X $($ c. $1348 \mathrm{C} \rightarrow \mathrm{T})$, in the CYP4V2 gene. Mutations were present in each of the 11 unrelated patients with BCD who were evaluated (table 1). Every patient was homozygous except one (patient 288-001), who was a presumed compound heterozygote. Two of the mutations, L173W and Q450X, are previously unreported; they were identified in one Japanese patient (patient 270) and in two Middle Eastern patients (patients 288-002 and 288-003), respectively. A representative pedigree and DNA sequence chromatogram for patients harbouring these novel mutations are presented in fig 1 .

The novel mutation L173W was interpreted as likely to be pathogenic for the following reasons. Firstly, leucine at position 173 is highly conserved among all six close eukaryotic homologues ranging from Homo sapiens to Arabidopsis thaliana (fig 2). Secondly, based on a structural analysis of the cytochrome p450 protein homologues CYPBM3 and CYP2C5, ${ }^{17}{ }^{18}$ L173 resides within an $\alpha$ helix presumably interacting with at least four other downstream helices. Thus, a change of the leucine at position 173 to tryptophan may result in a gross structural alteration possibly affecting the proper folding of the protein. Thirdly, the mutation was not found in an analysis of 100 Japanese normal controls.

The novel mutation Q450X was considered to be a pathogenic null mutation, as mRNA containing the predicted early stop codon would most likely be subject to nonsense mediated decay without being translated into a protein. Even if the translated protein is produced, it would lack amino acid sequences that are expected to coordinate the haem iron that binds substrate. ${ }^{10}$ This change was also not found among 90 healthy control individuals from North America.

Seven other Japanese patients with BCD were homozygotes for the IVS6-8_810delinsGC mutation that affected the IVS6 splice acceptor site. This mutation has been reported in other Japanese and Chinese BCD patients. ${ }^{10}$ Analysis of lymphocyte mRNA from patients indicated that the mutation causes in frame skipping of exon $7 .{ }^{10}$ Our Chinese patient had two mutations, IVS6-8_810delinsGC and H331P, and was a presumed compound heterozygote. There were no family members available for this to be confirmed by segregation analysis. Another BCD patient with the same combination of CYP4V2 alleles has been previously reported..$^{10}$

We categorised three missense changes, L22V (c.64C $\rightarrow \mathrm{G})$, $\mathrm{E} 259 \mathrm{~K}(\mathrm{c} .775 \mathrm{C} \rightarrow \mathrm{A})$, and R433Q (c.1328G $\rightarrow \mathrm{A})$, as likely nonpathogenic variants because they were all found in patients with other pathogenic recessive mutations in CYP4V2. An isocoding change A270A (c.810T $\rightarrow \mathrm{G}$ ) was found heterozygously in two patients and homozygously in one. This change, and the two likely non-pathogenic missense changes L22V and E259K have been previously reported in an SNP database (www.ncbi.nlm.nih.gov/SNP). Six intron changes $($ IVS $1-22 \mathrm{C} \rightarrow \mathrm{A}, \mathrm{IVS} 2+75 \mathrm{C} \rightarrow \mathrm{T}$, IVS3-40delCT, IVS4-25G $\rightarrow$ T, IVS6-7C $\rightarrow$ T, and IVS9+45A $\rightarrow G$ ) were found in patients with the other pathogenic recessive mutations in CYP $4 V 2$ described above. These changes were predicted not to affect RNA splicing, as assessed by online splice site prediction software (www.fruitfly.org/seq_tools/splice ${ }^{22}$ ), and were interpreted as non-pathogenic polymorphisms.

In the seven Japanese patients homozygous for the frequent IVS6-8_810delinsGC mutation, five non-pathogenic variants located between intron 1 and exon $6($ IVS $1-22 \mathrm{C} \rightarrow \mathrm{A}$, IVS2+75C $\rightarrow$ T, IVS3-40delCT, IVS4-25G $\rightarrow$ T, and c.775C $\rightarrow$ A) were observed in the homozygous state. The polymorphisms located in exon 1 (c.64C $\rightarrow \mathrm{G}$ ) and in intron 9 (IVS9+45A $\rightarrow \mathrm{G}$ ) were frequently identified in a screen of 50-100 normal Japanese subjects, and identified in some, but not all, of the BCD patients (fig 3).

The clinical characteristics of the BCD patients are summarised in table 1 . Seven patients complained of night blindness. Visual acuities of the 11 patients ranged from normal (1.0) to severely reduced (0.05). Eight patients had

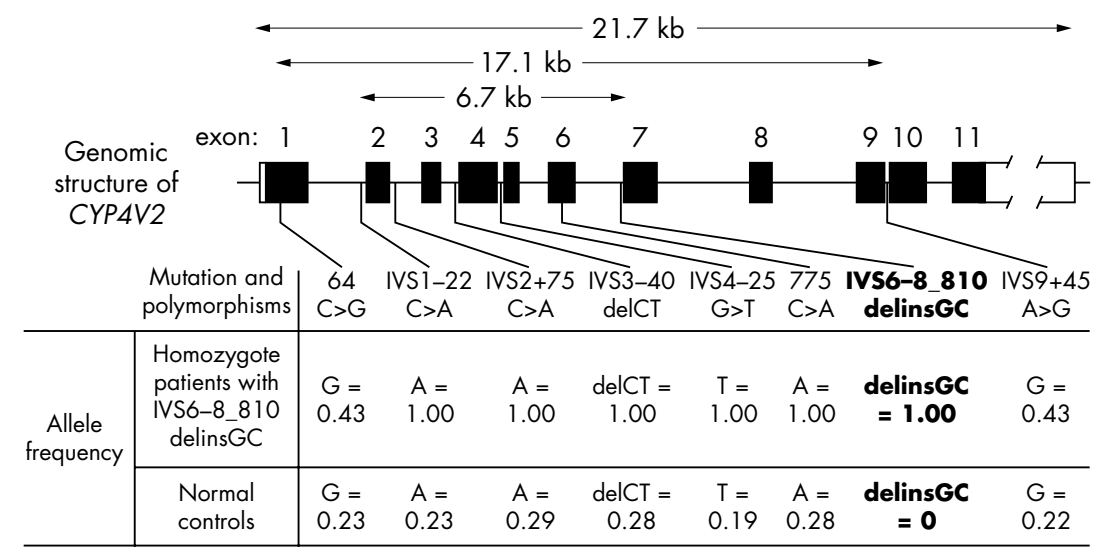

Figure 3 Distribution and allele frequency of the IVS6-8_810delinsGC mutation and polymorphisms identified in CYP4V2 gene among seven Japanese patients homozygous for the IVS6-8_810delinsGC mutation and 50 to 100 Japanese healthy control subjects. Aminoacid and genomic numbering are derived from XM_209612 and NC_000004, respectively. The disease causing mutation is highlighted in bold. 
A

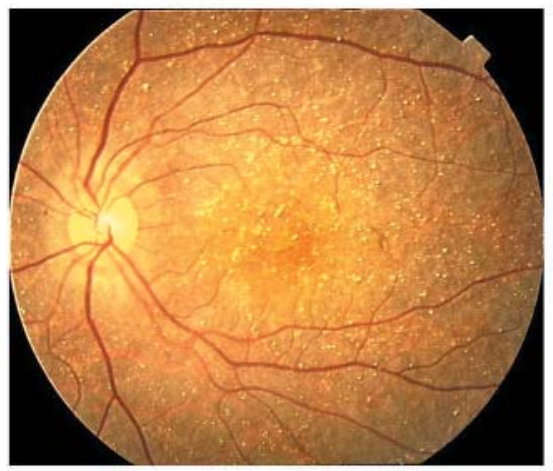

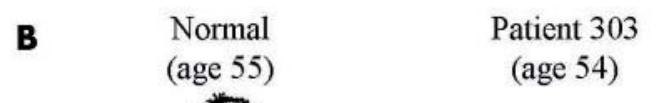

Patient 303
(age 54)

Patient 259

(age 52)

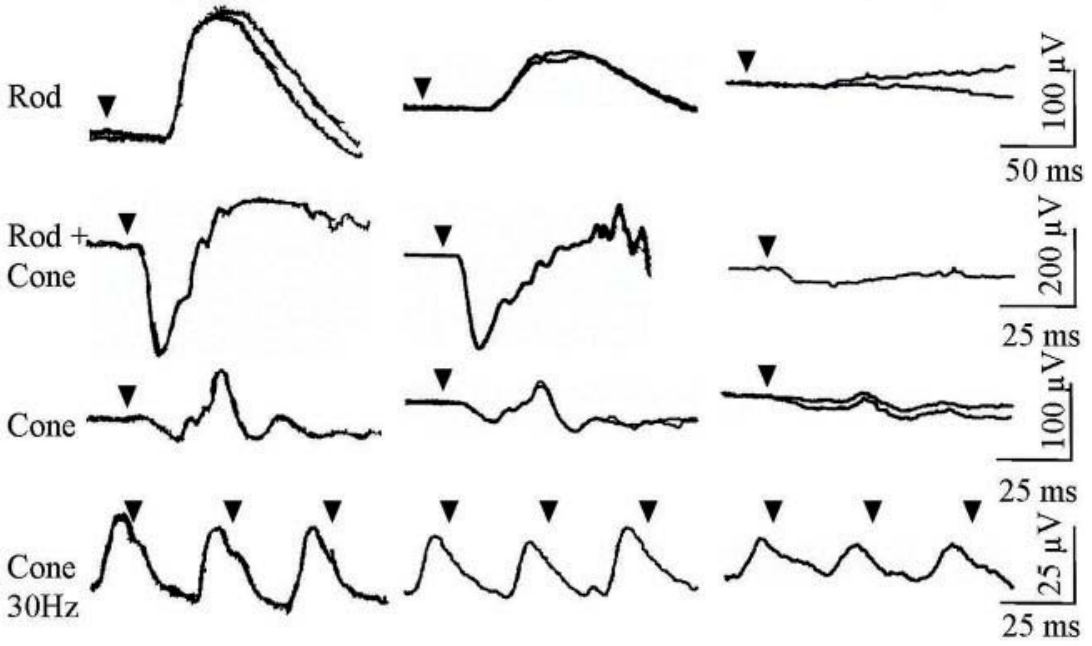

Figure 4 (A) Fundus photograph of the right eye of $B C D$ patient 260 with numerous typical crystalline deposits scattered throughout the fundus and diffuse atrophy of the retinal pigment epithelium. (B) Full field ERGs from a normal control (left) and two patients (patient 303 (middle) and patient 259 (right)) with the same CYP4V2 mutation (IVS6-8_810delinsGC) recorded at Nagoya University. From top: rod responses, rod plus cone responses, cone responses, and cone $30 \mathrm{~Hz}$ responses. Arrowhead indicates stimulus onset. The rod and the rod plus cone ERGs were recorded under dark adapted conditions, and the cone $30 \mathrm{~Hz}$ and cone ERGs under light adapted conditions. For each recording condition, a scale bar is shown at the right.

acuity in at least one eye better than 0.8. Slit lamp examination revealed peripheral corneal crystalline deposits in eight patients. Goldmann kinetic visual fields showed a central, a paracentral, or a ring scotoma in the patients (data not shown). All patients had characteristic numerous small retinal crystalline deposits concentrated in the posterior pole (a representative fundus photograph is presented in fig 4A). There were varying degrees of RPE atrophy and attenuation of the choriocapillaris at the posterior pole; these abnormalities sometimes extended to the midperiphery. Full field ERGs showed variable amounts of rod and cone function ranging from normal to severely reduced even among patients carrying the same mutation (representative ERGs dark-adapted are shown in fig 4B). The b-wave amplitudes for dark adapted single flash rod plus cone responses are summarised in table 1 .

\section{DISCUSSION}

We identified mutations in the CYP4V2 gene in all 11 unrelated patients we evaluated with typical BCD. These patients were of East Asian or Middle Eastern ancestry. A previous report showed that CYP $4 V 2$ mutations were found in 23 of 25 unrelated patients with BCD. ${ }^{10}$ Together with our results, it is apparent that defects in CYP4V2 account for most, if not all, cases of BCD.

In this screen, we found the homozygous IVS68_810delinsGC mutation in seven of eight unrelated Japanese patients with BCD. Consistent with the results in a previous study, which found the same mutation in seven of eight unrelated Japanese BCD patients, ${ }^{10}$ our findings indicate that this mutation is the major cause of BCD in Japan. The same mutation has been detected in seven of 10 unrelated Chinese patients, ${ }^{10}$ and in a Chinese patient in this study. Considering that this mutation is frequently identified in both Japanese and Chinese patients and that it has never been detected in BCD patients of other ethnic backgrounds, it is likely that all patients with this mutation descend from a single ancestor. This speculation would be supported by the observation that six closely linked polymorphic markers were identical in a homozygous state in all seven patients with the homozygous IVS6-8_810delinsGC mutation (fig 3). However, the founder of this mutation is probably a very distant ancestor because the region of the conserved linked markers is small, extending only $6.7-17.1 \mathrm{~kb}$ (fig 3). ${ }^{23}$

All patients with the CYP4V2 gene mutation shared similar characteristic fundus features, including crystalline deposits and RPE atrophy. However, the reduction of visual acuity and the amount of visual field loss varied among patients. Full field ERGs, reflecting overall retinal function, showed remarkable variability in amplitudes, as has been previously reported. ${ }^{24}$ Our results indicate that severity differed even among patients at similar ages with the same IVS68 810delinsGC mutation, raising the possibility that factors besides the primary gene defect influence the course of the retinal disease.

It has been reported that corneal crystalline deposits are present in one quarter to one third of patients with BCD. ${ }^{5}$ We observed them in eight of 11 patients by slit lamp examination. However, as corneal crystalline deposits are very subtle, and in some patients can be detected clearly only by specular microscopy, ${ }^{25}$ the true number of patients with corneal deposits may be higher.

\section{ACKNOWLEDGEMENTS}

This study received support from a grant in aid for Scientific Research from the Ministry of Education, Culture, Sports, Science, and Technology of Japan (C16591746), grants EY08683, EY1404, and EY00169 from the National Eye Institute of the United States, and the Foundation Fighting Blindness, Owings Mills, Maryland.

\section{Authors' affiliations}

J Lin, K M Nishiguchi, M Nakamura, Y Miyake, Department of Ophthalmology, Nagoya University Graduate School of Medicine, 65 Tsuruma-cho, Showa-ku, Nagoya 466-8550, Japan

K M Nishiguchi, E L Berson, T P Dryja, Ocular Molecular Genetics Institute and the Berman-Gund Laboratory for the Study of Retinal Degenerations, Massachusetts Eye and Ear Infirmary, Harvard Medical School, Boston, MA 02114, USA 
Competing interest: none declared

The first two authors contributed equally to this work.

Correspondence to: Dr M Nakamura, Department of Ophthalmology, Nagoya University Graduate School of Medicine, 65 Tsuruma-cho, Showa-ku, Nagoya 466-8550, Japan; makonaka@med.nagoya-u.ac.jp

Received 18 November 2004

Revised version received 12 January 2005

Accepted for publication 13 January 2005

\section{REFERENCE}

1 Bietti GB. Ueber familiaeres vorkommen von "retinitis punctata albescens" (verbunden mit "dystrophia marginalis cristallinea corneae"), glitzern des glaskoerpers und anderen degenerativen augenveraenderungen. Klin Mbl Augenheilk 1937;99:737-57.

2 Bagolini B, loli-Spada G. Bietti's tapetoretinal degeneration with marginal corneal dystrophy. Am J Ophthalmol 1968;65:53-60.

3 Bernaver W, Daicker B. Bietti's corneal-retinal dystrophy. A 16-year progression. Retina 1992;12:18-20.

4 Welch RB. Bietti's tapetoretinal degeneration with marginal corneal dystrophy crystalline retinopathy. Trans Am Ophthalmol Soc 1977;75:164-79.

5 Kaiser-Kupfer MI, Chan CC, Markello TC, Crawford MA, Caruso RC, Csaky KG, Guo J, Gahl WA. Clinical biochemical and pathologic correlations in Bietti's crystalline dystrophy. Am J Ophthalmol 1994;1 18:569-82.

6 Wilson DJ, Weleber RG, Klein ML, Welch RB, Green WR. Bietti's crystalline dystrophy. A clinicopathologic correlative study. Arch Ophthalmol 1989;107:213-21.

7 Hu DN. Ophthalmic genetics in China. Ophthal Paediat Genet 1983;2:39-45

8 Yagasaki K, Miyake Y. (Crystalline retinopathy). Nippon Ganka Gakkai Zasshi 1986;90:711-19.

9 Jiao X, Munier FL, Iwata F, Hayakawa M, Kanai A, Lee J, Schorderet DF Chen MS, Kaiser-Kupfer M, Hejtmancik JF. Genetic linkage of Bietti crystallin corneoretinal dystrophy to chromosome 4q35. Am J Hum Genet 2000;67:1309-13.

10 Li A, Jiao X, Munier FL, Schorderet DF, Yao W, Iwata F, Hayakawa M, Kanai A, Shy CM, Alan LR, Heckenlively J, Weleber RG, Traboulsi El, Zhang Q, Xiao X, Kaiser-Kupfer M, Sergeev YV, Hejtmancik JF. Biett crystalline corneoretinal dystrophy is caused by mutations in the novel gene CYP4V2. Am J Hum Genet 2004;74:817-26.

11 Lee J, Jiao X, Hejtmancik JF, Kaiser-Kupfer M, Chader GJ. Identification, isolation, and characterization of a $32-\mathrm{kDa}$ fatty acid-binding protein missing from lymphocytes in humans with Bietti crystalline dystrophy (BCD). Mol Genet Metab 1998;65: 143-54

12 Nakamura M, Hotta Y, Tanikawa A, Terasaki H, Miyake Y. A high association with cone dystrophy in fundus albipunctatus caused by mutations of the RDH5 gene. Invest Ophthalmol Vis Sci 2000;41:3925-32.
13 Nakamura $M$, Ito S, Terasaki $H$, Miyake $Y$. Novel CACNA1F mutations in Japanese patients with incomplete congenital stationary night blindness. Invest Ophthalmol Vis Sci 2001;42:1610-16.

14 Andreasson SO, Sandberg MA, Berson EL. Narrow-band filtering for monitoring low-amplitude cone electroretinograms in retinitis pigmentosa. Am J Ophthalmol 1988; 105:500-3.

15 Reichel E, Bruce AM, Sandberg MA, Berson EL. An electroretinographic and molecular genetic study of X-linked cone degeneration. Am J Ophthalmol 1989; 108:540-7.

16 Berson EL, Gouras P, Gunkel RD. Rod responses in retinitis pigmentosa, dominantly inherited. Arch Ophthalmol 1968;80:58-67.

17 Lee C. Predicting protein mutant energetics by self-consistent ensemble optimization. J Mol Biol 1994;236:918-39.

18 Lee C, Subbiah S. Prediction of protein side-chain conformation by packing optimization. J Mol Biol 1991;217:373-88.

19 Kawai J, Shinagawa A, Shibata K, Yoshino M, Itoh M, Ishii Y, Arakawa T, Hara A, Fukunishi Y, Konno H, Adachi J, Fukuda S, Aizawa K, Izawa M, Nishi K, Kiyosawa H, Kondo S, Yamanaka I, Saito T, Okazaki Y, Gojobori T, Bono H, Kasukawa T, Saito R, Kadota K, Matsuda H, Ashburner M, Batalov S, Casavant T, Fleischmann W, Gaasterland T, Gissi C, King B, Kochiwa H, Kuehl P, Lewis S, Matsuo Y, Nikaido I, Pesole G, Quackenbush J, Schriml LM, Staubli F, Suzuki R, Tomita M, Wagner L, Washio T, Sakai K, Okido T, Furuno $M$, Aono $H$, Baldarelli $R$, Barsh $G$, Blake J, Boffelli D, Bojunga N Carninci P, de Bonaldo MF, Brownstein MJ, Bult C, Fletcher C, Fujita M, Gariboldi M, Gustincich S, Hill D, Hofmann M, Hume DA, Kamiya M, Lee NH, Lyons P, Marchionni L, Mashima J, Mazzarelli J, Mombaerts P, Nordone P, Ring B, Ringwald M, Rodriguez I, Sakamoto N, Sasaki H, Sato K, Schonbach C, Seya T, Shibata Y, Storch KF, Suzuki H, Toyo-oka K, Wang KH, Weitz C, Whittaker C, Wilming L, Wynshaw-Boris A, Yoshida K, Hasegawa Y, Kawaji H, Kohtsuki S, Hayashizaki Y. Functional annotation of a full-length mouse cDNA collection. Nature 2001:409:685-90.

20 Piano F, Schetter AJ, Morton DG, Gunsalus KC, Reinke V, Kim SK Kemphues KJ. Gene clustering based on RNAi phenotypes of ovary-enriched genes in C. elegans. Curr Biol 2002;12:1959-64.

21 Walhout AJ, Reboul J, Shtanko O, Bertin N, Vaglio P, Ge H, Lee H, DoucetteStamm L, Gunsalus KC, Schetter AJ, Morton DG, Kemphues KJ, Reinke V, Kim SK, Piano F, Vidal M. Integrating interactome, phenome, and transcriptome mapping data for the $C$. elegans germline. Curr Bio 2002; 12:1952-8.

22 Reese MG, Kulp D, Tammana H, Haussler D. Genie-gene finding in Drosophila melanogaster. Genome Res 2000;10:529-38.

23 Risch N, de LD, Ozelius L, Kramer P, Almasy L, Singer B, Fahn S, Breakefield X, Bressman S. Genetic analysis of idiopathic torsion dystonia in Ashkenazi Jews and their recent descent from a small founder population. Nat Genet 1995;9:152-9.

24 Yanagi Y, Tamaki Y, Takahashi H, Sekine H, Mori M, Hirato T, Okajima O. Clinical and functional findings in crystalline retinopathy. Retina 2004;24:267-74

25 Takikawa C, Miyake Y, Yamamoto S. Re-evaluation of crystalline retinopathy based on corneal findings. Folia Ophthalmol Jpn 1992;43:969-78. 